\title{
Leakage current correction in quasi-static $C-V$ measurements
}

\author{
J. Schmitz, M. H. H. Weusthof and A. J. Hof \\ MESA+ Research Institute, University of Twente \\ P.O. Box 217, 7500 AE Enschede, The Netherlands \\ E-mail: J.Schmitz@utwente.nl
}

Abstract - The gate current in a MOS structure can deform the result of a quasi-static capacitance-voltage measurement. In this paper, several correction methods are presented and discussed to compensate for this effect. Limitations of all methods are quantified and workarounds are proposed.

\section{INTRODUCTION}

The Quasi-Static Capacitance-Voltage (QS-CV) measurement $[1,2]$ (also called linear ramp $C-V$ ) is a powerful characterization method for quantifying the properties of a MOS capacitor. As compared to a highfrequency $C-V$ measurement, its key benefit is the ability to obtain information on slow interface states at the semiconductor-dielectric interface. However, with the ongoing scaling of MOS transistors, the dielectrics are becoming ultra-thin and hence show a considerable tunnel current. This tunnel current is indistinguishable from the quasi-static charging current in a traditional QS-CV measurement. Gate dielectrics with a thickness around $5 \mathrm{~nm}$ (and thinner) therefore exhibit deformed QS-CV characteristics.

High-K dielectrics in the EOT-range foreseen in future CMOS processes also show a tunnel current that is considerable for a QS.CV measurement. For the characterization of slow interface traps in a high-K MOS capacitor, the QS-CV method must be adapted to compensate for the gate leakage current. For reasons of convenience, in this paper we study such compensation scenarios using measurements on traditional $\mathrm{SiO}_{2}$ dielectrics.

Several off-line correction methods can be applied on deformed $C-V$ characteristics; alternatively, specialized hardware can be used that compensates for gate leakage. In this paper we present and evaluate various methods of leakage current correction in QS-CV measurements.

\section{EXPERIMENTAL DETAILS}

MOS capacitors and transistors with $5 \mathrm{~nm}$ gate dielectrics were manufactured using a standard $0.18 \mu \mathrm{m}$ NMOS process flow, on $10 \Omega \mathrm{cm}$ wafers. The process features STI field isolation, thermal gate oxidation and $\mathrm{n}^{+}$polysilicon gates and backside metallisation. Measurements were carried out on a $0.81 \mathrm{~mm}^{2}$ capacitor with $\mathrm{n}^{+}$diffusion edge. Capacitors with $25 \mathrm{~nm}$ (thermally grown) gate dielectrics were fabricated on $5-10 \Omega \mathrm{cm}$ wafers with $1-\mu \mathrm{m}$ sputtered aluminum gates and backside metallisation. The sintering anneal was purposely left out on some wafers to achieve a high level of interface states. Measurements were carried out on a $1-\mathrm{mm}^{2}$ metaloxide-silicon capacitor without diffusion edge.
A Cascade probe station and Agilent's $4156 \mathrm{C}$ were used for capacitance-voltage measurements. Data were verified with the HP 4140B (and no deviations were found). The guidelines for accurate $C-V$ measurements on the $4156 \mathrm{C}$ in $[3,4]$ were followed where possible.

\section{GATE LEAKAGE CORRECTION ALGORITHMS}

To correct for gate leakage, the gate current must first be quantified as a function of gate voltage. Various options are at hand [5]:

- Quantification of the gate current using a separate dc $I-V$ measurement, with e.g. any parameter analyzer;

- A measurement of QS-CV at various sweep rates to distinguish leakage from quasi-static current;

- Leakage current measurement during temporary interruptions of the QS-CV measurement.

All three methods have been evaluated and they are discussed in the following subsections.

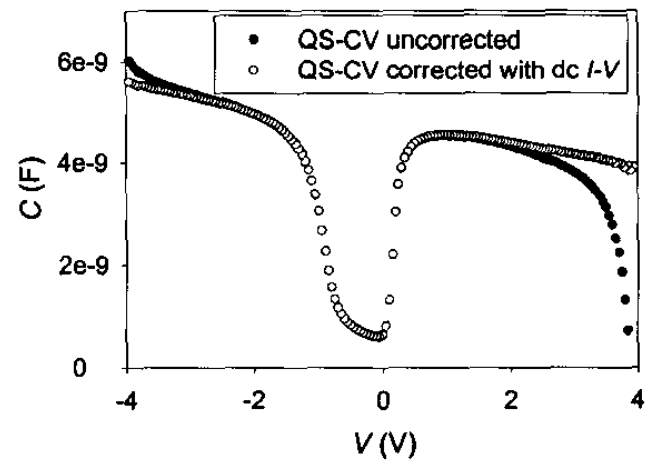

Figure 1: Quasi-static $C-V$ measurements in the presence of gate leakage, as measured on an NMOS capacitor with $5 \mathrm{~nm}$ gate oxide. Also shown is the curve after correction by tunnel current, separately measured using a Parameter Analyzer.

\section{Correction with dc I-V data}

A typical QS-CV curve suffering from gate leakage is shown in Figure 1. The strong inversion capacitance is underestimated at the point where leakage current becomes significant. A dc $J-V$ measurement of the gate leakage ( $\left.I_{\text {tunnel }}\right)$, shown in Figure 2, can be used to compensate for this leakage (as depicted in Figure 1). The quasi-static charging current $I_{\mathrm{QS}}$ is simply obtained using $I_{\text {total }}=I_{\mathrm{QS}}+I_{\text {tunnel }}$. This approach relies first of all on the correct procedure for the dc I-V measurement: the quasi-static charging current should not 
contribute to the measured dc current. Sufficient settling time must therefore be chosen, also considering the response time of slow interface traps. The method naturally becomes inaccurate when $I_{\text {tunnel }} \gg I_{\mathrm{QS}}$, due to limited instrument precision.

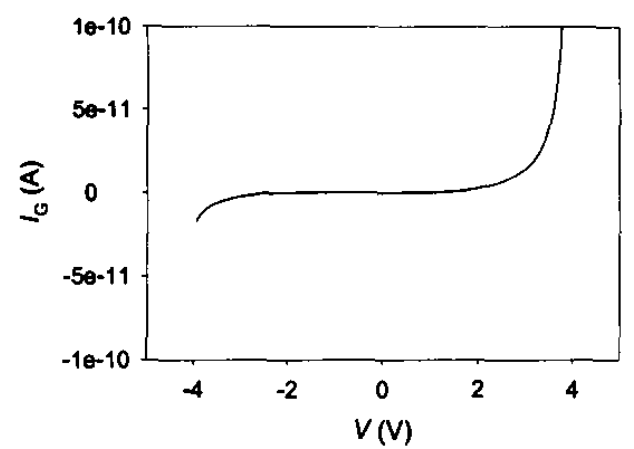

Figure 2: current-voltage characteristic of the capacitor presented in figure 1 .

\section{Measurements at various sweep rates}

The quasi-static charging current $I_{\mathrm{QS}}$ is directly proportional to the voltage sweep rate, $d V / d t$. Since $I_{\text {tunnel }}$ does not depend on $d V / d t$, a QS-CV measurement at various sweep rates can separate $I_{\mathrm{QS}}$ from $I_{\text {tunnel. }}$. Figure 3 shows how such an approach can be used to quantify both $I_{\mathrm{QS}}$ and $I_{\text {tunnel. }}$. The real capacitance $C$ relates to the measured capacitance $C_{\mathrm{m}}$ as

$$
C=C_{\mathrm{m}}-I_{\text {tannel }}(d V / d t)^{-1}
$$

and with measurements of $C_{\mathrm{m}}$ at two values of $d V / d t$ one obtains two equations with two unknowns ( $C$ and $\left.I_{\text {tunnel }}\right)$. A complication with this method is the fact that interface traps have a characteristic response time, and slow traps may be missed when the larger sweep rate is used, leading to possible misinterpretation of the results in the depletion region of the $C-V$ curve. Fortunately, this is the region where gate leakage is at its lowest, so the leakage correction procedure does not need to be applied in the deep depletion part of the curve.

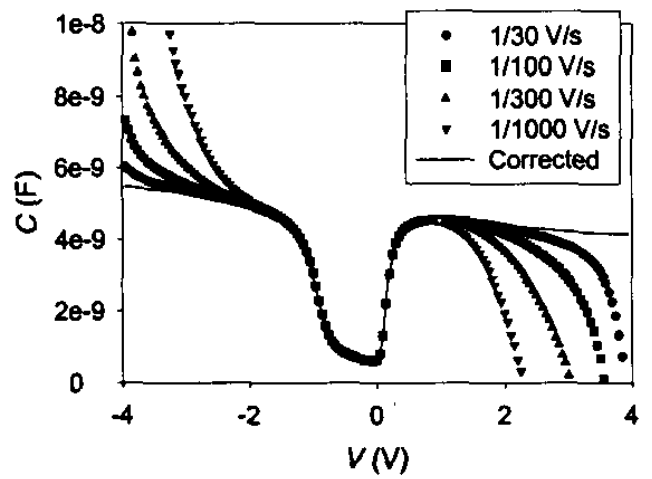

Figure 3: QS-CV with gate leakage measured at various sweep rates; and the corrected QS-CV curve using the fastest two sweeps.
A special case of the two-sweep method is the forwardbackward sweep, where a QS-CV curve is measured with $+d V / d t$ and $-d V / d t$, respectively. This approach is quick and simple, and works well as long as the dielectric layer contains negligible mobile charge. This method is therefore not recommended for characterization of high- $\mathrm{K}$ layers.

\section{A periodically interrupted voltage sweep}

Leakage current and quasi-static charging current can be separately measured with the same current meter using various algorithms. For instance, the ramped voltage sweep can be interrupted at fixed voltages to allow the measurement of the steady state leakage. The separation of the transient current and the steady current can be hardwired into a $C-V$ measurement instrument.

The Agilent 4156C parameter analyzer offers automatic gate leakage correction on QS-CV measurements using such an approach. Indeed, this method yields good results when gate leakage occurs: see Figure 4.

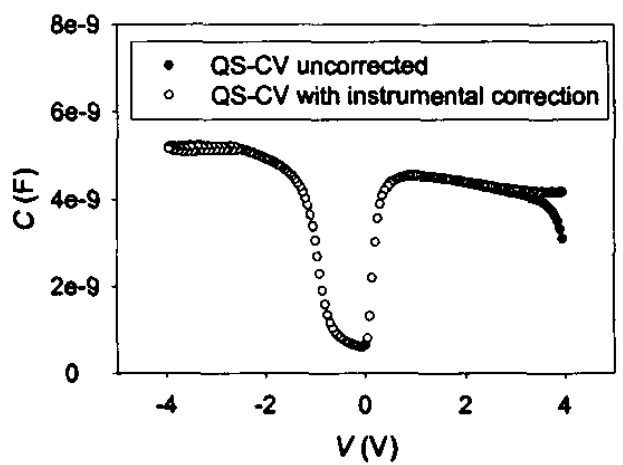

Figure 4: The effect of gate leakage correction on a leaky MOS capacitor with $5 \mathrm{~nm}$ gate dielectric, as obtained with the Agilent $4156 \mathrm{C}$ automatic leakage current correction function.

But there is an important pitfall when automatic leakage correction is applied on devices with a significant interface state density. In deep depletion, part of the flowing current is due to the filling of these interface states. We found that this current is not correctly taken into account in the leakage correction algorithm. As a result, the leakage current correction can give rise to an artificial increase or decrease of the apparent number of interface states.

The effect is illustrated with Figure 5. The presented measurements were carried out on 25-nm dielectrics, to exclude any gate leakage effects. (It is of course useless to apply the instrument's gate leakage correction on a perfectly insulating dielectric, but here it is used on purpose, to unambiguously connect the discovered artifact to interface states.) The capacitance shift introduced by the leakage correction algorithm can be larger than $10 \%$ in the deep depletion regime, leading to a systematic underestimate of the interface state density.

The artifact shown in Figure 5 is observed for a wide range of settings on the $4156 \mathrm{C}$. We separately varied delay time, $d V$ and $d t$ (for definitions, see [3]). The impact of the latter two parameters on the shift in capacitance is shown in Figures 6 
and 7. The capacitance at $V=-0.4 \mathrm{~V}$ was taken as an indicator of the overall shift in deep depletion.

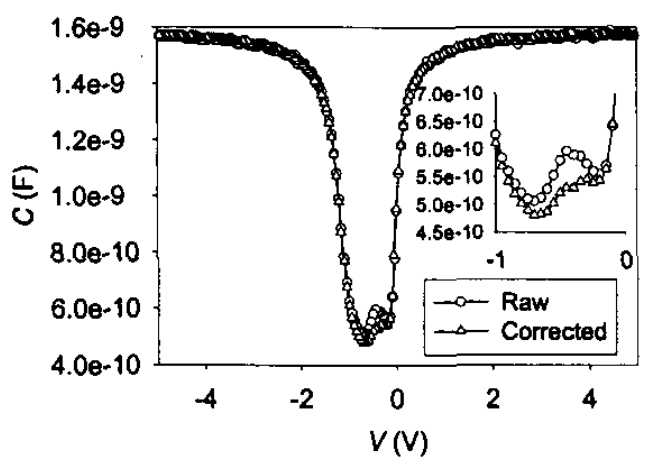

Figure 5: A $C-V$ characteristic with significant interface traps. The normal QS-CV characteristics are shown ('raw') as well as those after correction for (non-existing) gate leakage current ('corrected'). The current flowing to supply charge to the traps is misinterpreted as gate leakage current and the capacitance curve is incorrectly adjusted.

It is clear from Figures 6 and 7 that the apparent loss of capacitance in depletion is observed over a wide range of instrumental settings. The effect appears to get stronger with smaller $d V / d t$ values. We attribute this to the relatively high density of very slow traps as compared to faster traps. The effect of course increases when the interface trap density is higher.

It can be concluded that the automatic leakage current correction algorithm of the $4156 \mathrm{C}$ has the side effect of suppressing the interface state signature; because the interface charge trapping current is partly misinterpreted as gate leakage current.

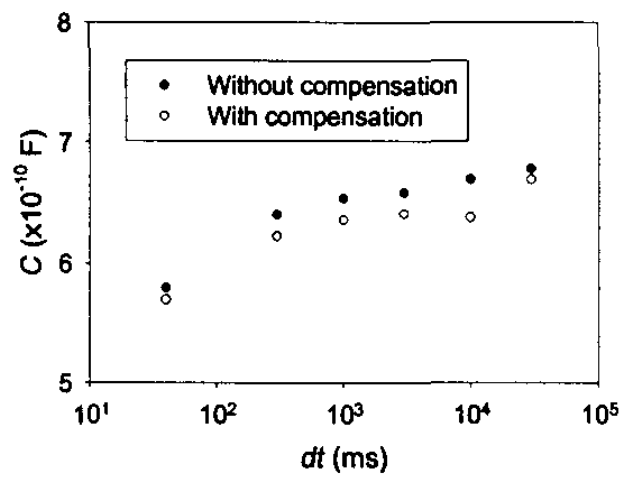

Figure 6: the effect of the choice of $d t$ on the apparent capacitance loss in depletion. The graph shows the depletion capacitance at -0.4 $\mathrm{V}$ as measured ('without compensation') and after gate leakage correction ('with compensation'). The measurement was carried out on the capacitor of Figure 5. Delay time was $100 \mathrm{~ms}, d V$ was 100 $\mathrm{mV}$.

Leakage current correction can introduce artifacts and should therefore be used wisely (and analyzed with somewhat extra care). Still, such a correction does enable the reconstruction of the capacitance-voltage characteristic when moderate gate leakage is present. It typically works well as long as $I_{\mathrm{g}}<I_{\mathrm{QS}}$. All of the presented correction procedures will fail when the tunnel current is much larger than the quasi-static charging current, because of limited instrument precision and gate current noise.

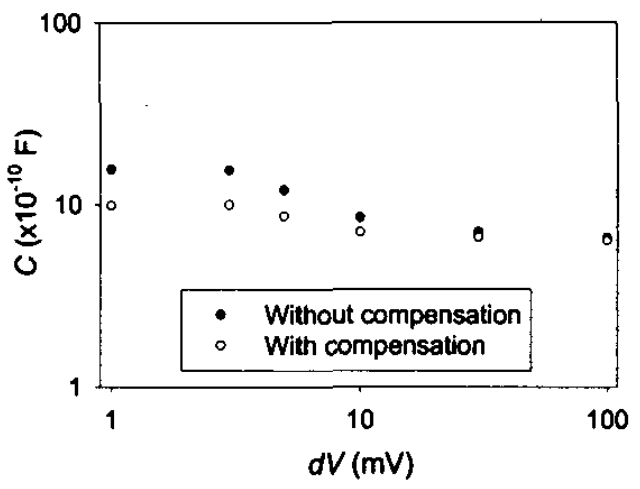

Figure 7: the effect of the choice of $d V$ on the apparent capacitance loss in depletion, as in Figure 6. Delay time was $100 \mathrm{~ms}$, $d t$ was $1 \mathrm{~s}$.

\section{CONCLUSIONS}

Three approaches are presented for the correction of gate leakage current in quasi-static $\mathrm{C}-\mathrm{V}$ measurements. Correction with a separate dc $I-V$ measurement is straightforward and effective. Retrieving the quasi-static capacitance and gate leakage current from measurements at two sweep rates can be as precise, be it that the normal precaution must be made that the sweep rate is not too high. Slow interface states may be identified only at the lower sweep rate, which complicates the analysis in deep depletion. Automatic correction is effective and probably the most efficient, but it may introduce artifacts: the interface trap density can be significantly underestimated in the corrected $C-V$ curve.

\section{ACKNOWLEDGEMENTS}

The wafers with $5 \mathrm{~nm}$ gate dielectrics were kindly provided by Philips Research. The work of one of the authors (Hof) is financially supported by Philips Semiconductors. We would like to thank Bill Verzi for fruitful discussions.

\section{REFERENCES}

[1] E. H. Nicollian and J. R. Brews, MOS (Metal Oxide Semiconductor) physics and technology, John Wiley \& Sons, New York 1982.

[2] D. K. Schroder, Semiconductor Material and Device Characterization, John Wiley \& Sons, New York 1998.

[3] Agilent 4155C/4156C User's Guide Volume 2: measurement and analysis.

[4] Agilent application note 4156-10: evaluation of gate oxides using a voltage step quasi-static CV method.

[5] J. Schmitz and L. F. Tiemeijer, Trends in CMOS device characterization (invited), INFOS 2003. 